SECTION 21. Pedagogy. Psychology. Innovations in the field of education.

Shevtsov Alexandr Nikolayevich

candidate of technical Sciences,

President, Theoretical \& Applied Science, LLP, associate Professor of the Department «Applied mathematics» Taraz State University named after M.Kh. Dulati, Kazakhstan

Kenysheva Akbota Yeralykyzy

3 year student, the specialty "Mathematics"

Taraz State University named after M.Kh. Dulati, Kazakhstan

\title{
CONTEMPORARY APPROACHES TO THE TEACHING ON THE EXAMPLE OF LABORATORY WORKS ON THE THEORY OF GRAPHS
}

This article describes methods of teaching and laboratory works on discipline of graph theory.

Keywords: graphs, laboratory work, methodology.

\section{СОВРЕМЕННЫЕ ПОДХОДЫ К ПРЕПОДАВАНИЮ НА ПРИМЕРЕ ЛАБОРАТОРНЫХ РАБОТ ПО ТЕОРИИ ГРАФОВ}

B данной статье описывается методика преподавания и проведения лабораторных работ по дисциплине - теория графов.

Ключевые слова: графы, лабораторная работа, методика.

Почти во всех дисциплинах изучаемых в настоящее время - предусмотренны часы на лобораторные работы. Лабораторные по математике могут проводиться как без использования компьютеров, но это скорее стоит отнести к практическим занятиям, так и с использованием. Обязательным атрибутом лабораторного занятия - является методические пособия для проведения лабораторной работы. Таких пособий по математике сейчас достаточно мало.

Рассмотрим пример разработки и проведения лабораторной работы по дисциплине «Теория графов» для 3 курса университета специальности «Математика». За основе возьмем РУП данной специальности, а в качестве методической основы - пособие Кирсанова М.Н. [1].

Тема занятия - Методы кодирования графов. Цель занятия: научить студентов самостоятельно использовать различные виды кодировок для кодирования графов. На лабораторную отводится 3 часа. Лабораторная проводилась в компьютерной аудитории на 20 компьютеров. В течении первого занятия студентами изучались следующие кодировки графов: Десятичная кодировка, Кодировка Прюфера, Код Гапфа (табл.1). В течение второго занятия студенты выполняли самостоятельную работу по построению графов и вычисления соответствующих изученных кодировок для них. Результат самостоятельной работы одного из студентов приведен в табл.2.

Таблица 1

\section{Вычисление кода Прюфера}



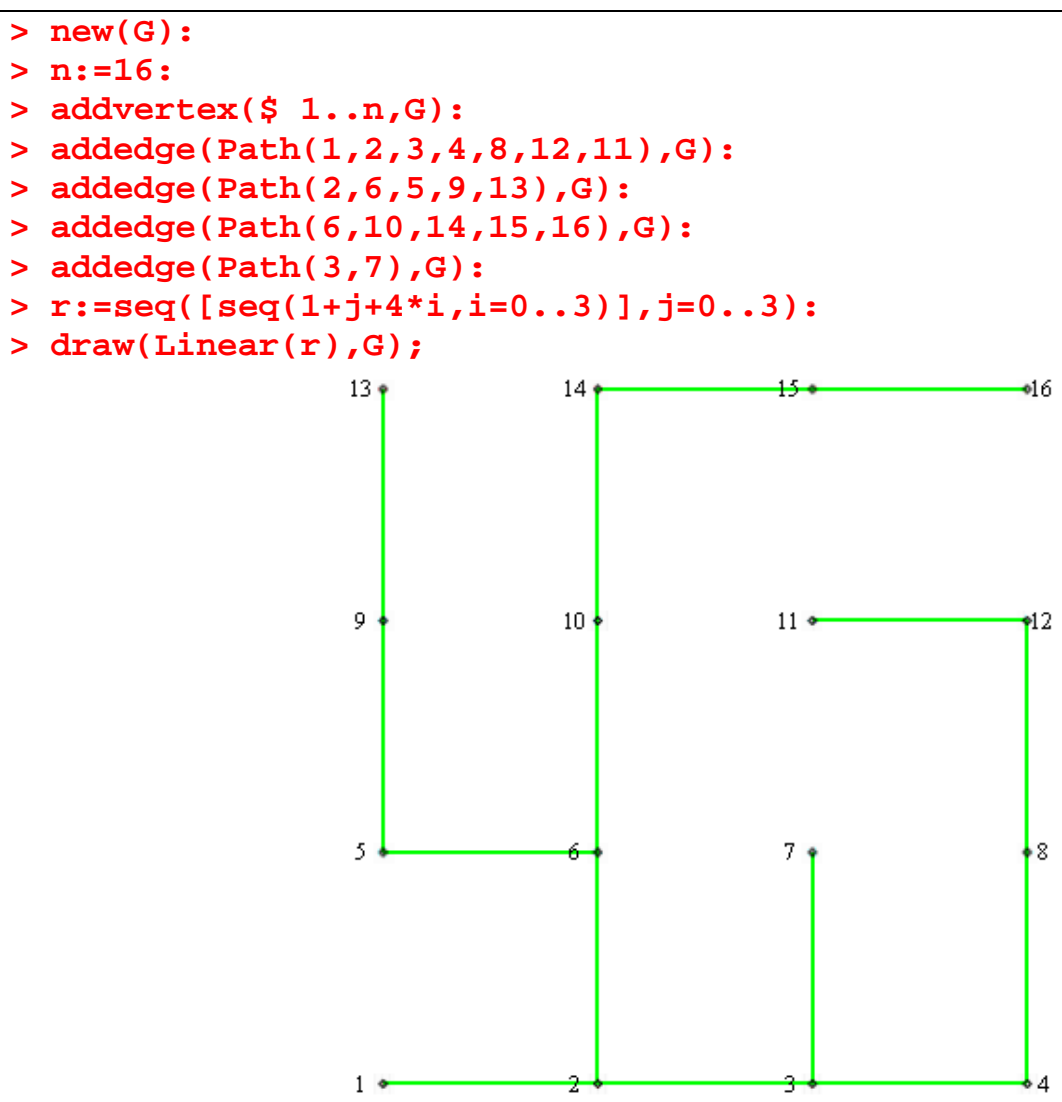

$>$

$>T:=[0 \$(n-2)]:$

for $i$ to $(n-2)$ do

$>$ mindegree $(G, \operatorname{sm}[i])$ :

$>\mathrm{z}:=\operatorname{sm}[\mathrm{i}]$ :

$>$ z1:=departures $(z, G)$ :

$>\mathrm{T}[\mathrm{i}]:=\mathrm{z} 1[1]$ :

$>\operatorname{delete}(\{z\}, G)$ :

$>$ od:

$>\mathbf{T}$;

Код Прюфера:

$[2,3,12,8,4,3,2,6,9,5,6,10,14,15]$

\section{Результат самостоятельной работы}

Таблица 2

$>$ restart;

$>$ with(networks):

$>\operatorname{new}(G)$ :

$>\mathrm{n}:=36$ :

$>$ addvertex $(\$ 1 . . n, G)$ :

$>r:=\operatorname{seq}([\operatorname{seq}(1+j+6 * i, i=0.5)], j=0 . .5):$

$>$ addedge (Path $(10,11,12,6,5,4,3,9,15,16,17,23,22,21,20,26,25,31), G)$ :

$>$ addedge $(\operatorname{Path}(18,17,23,24,30,36,35,34,33,32), \mathrm{G})$ :

$>$ addedge $(\operatorname{Path}(35,29,28,27), \mathrm{G})$ :

$>$ addedge $(\operatorname{Path}(19,20,14,8,7,13), \mathrm{G})$ :

$>\operatorname{addedge}(\operatorname{Path}(8,2), \mathrm{G})$ :

$>\operatorname{addedge}(\operatorname{Path}(7,1), \mathrm{G})$ :

$>\operatorname{draw}(\operatorname{Linear}(r), \mathrm{G})$; 


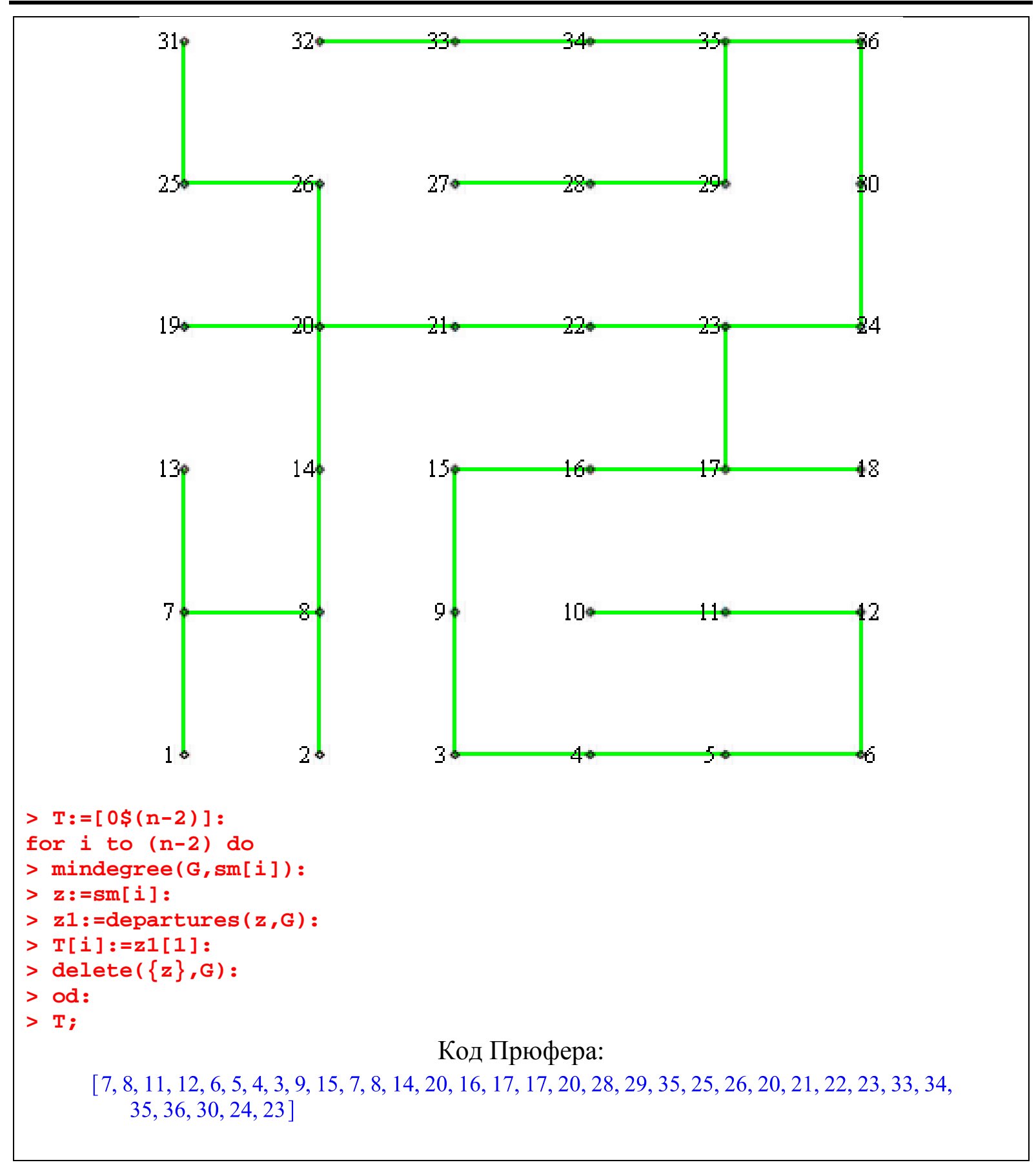

Использование современных программных продуктов, и компьютеров - является неотъемлимой частью современного образования, способствует лучшему усвоению студентами материала дисциплины, а также вызывает у них интерес и стремление к учебе.

\section{Литература}

1. Кирсанов М.Н. Графы в Maple. Задачи, алгоритмы, программы. -М.: Издательство ФИЗМАТЛИТ, 2007. -168c. 